\title{
The prevalence of insulin refusal and psychological insulin resistance among Korean patients with type 2 diabetes mellitus
}

\author{
Youngshin Song ${ }^{1}$, Bon Jeong $\mathrm{Ku}^{2}$, Jeonghwa $\mathrm{Cho}^{3}$, Younghee $\mathrm{Jun}^{4}$, Bohyun $\mathrm{Kim}^{1}$, Soohyun $\mathrm{Nam}^{5}$ \\ ${ }^{1}$ Chungnam National University College of Nursing, Daejeon, Republic of Korea; ${ }^{2}$ Chungnam National University College of Medicine, Daejeon, \\ Republic of Korea; ${ }^{3}$ Daejeon Institute of Science and Technology Department of Nursing, Daejeon, Republic of Korea; ${ }^{4}$ Wonkwang Health Science \\ University Department of Nursing, Wonkwang, Republic of Korea; ${ }^{5}$ Yale University, School of Nursing, West Haven, CT, USA \\ Correspondence to: Bon Jeong Ku. Department of Internal Medicine, Chungnam National University College of Medicine, 266 Munhwa-ro, Jung-gu, \\ Daejeon 35015, Republic of Korea. Email: bonjeong@cnu.ac.kr.
}

\begin{abstract}
Background: This study investigated the prevalence of insulin refusal and compared the level of psychological insulin resistance (PIR) with the intention to accept insulin therapy among Korean patients with type 2 diabetes.

Methods: Type 2 diabetes patients $(n=1,209)$ were recruited from inner city outpatient diabetes clinics in 2016. Patient demographic, insulin recommendation, diabetes treatment type, intention to receive insulin therapy, and PIR information was collected using self-reports. The patients were divided into three groups: those who refused insulin therapy $(n=184)$, those who accepted insulin therapy $(n=331)$, and those not recommended for insulin therapy $(\mathrm{n}=694)$. Descriptive statistics were used to calculate the prevalence of insulin refusal; $t$-tests and analyses of variance were used to compare demographics with PIR.
\end{abstract}

Results: The insulin refusal rate was $35.7 \%$. The duration of diabetes, numbers of diabetes-related complications, and glycated hemoglobin levels were significantly lower in the group not recommended for insulin therapy than refused and accepted insulin groups. The level of PIR in insulin refusal group was significantly lower among patients agreeing to receive insulin therapy within 3 months than among those refusing insulin therapy.

Conclusions: The prevalence of insulin refusal in Korea is not low, and people who refuse insulin therapy have high levels of PIR. Strategies to reduce PIR levels are needed.

Keywords: Diabetes mellitus; insulin; medication adherence; treatment refusal

Submitted Aug 23, 2019. Accepted for publication Nov 05, 2019.

doi: $10.21037 /$ atm.2019.11.77

View this article at: http://dx.doi.org/10.21037/atm.2019.11.77

\section{Introduction}

Insulin therapy in diabetes is important to reduce hyperglycemia. Patients with type 2 diabetes receiving intensive insulin therapy can achieve optimal glycemic control, resulting in delaying or even preventing microand macro-complications, such as cardiovascular disease (1). Guidelines on insulin therapy (1) recommend that insulin be prescribed when blood glucose level remains uncontrolled after treatment with a combination regimen ( 2 or 3 kind medications). Despite the importance of insulin therapy, many patients with type 2 diabetes are reluctant toward or fearful of insulin therapy (2). This negative perception of insulin therapy is referred to as "psychological insulin resistance" (PIR) $(3,4)$. Patients with PIR experience negative self-perception and worry about insulin-related side effects, social stigma, and required lifestyle adaptations (2). These worries, in turn, lead to a delay in treatment and tend to negatively affect outcomes.

A growing number of insulin-refusing type 2 diabetes patients increases the risk of a significant number of patients not achieving glucose control, facing diabetes-related complications, and impaired quality of life (5). Recent studies report insulin therapy refusal rate among insulinnaïve patients with type 2 diabetes in the range of $20-70 \%$ 
(6-12), with Asian populations more likely to refuse insulin than non-Asian populations (13). In fact, insulin refusal rate was reported as $70.6 \%$ in Singapore (6) and $42.5 \%$ in Bangladesh (8), compared to $33.0 \%$ in the United States (8). Moreover, insulin refusal rates are higher among lowincome and racial minority populations, regardless of country of origin or residence (11).

Attitudes and beliefs of patients from Korea regarding insulin therapy may differ from those of patients from other cultures or countries. Specifically, diabetes management, including adherence to insulin therapy, is not always seen as high priority among the Korean population, with family obligations or financial comforts often making top of the list (14-16). This suggests that the differences in insulin refusal rates may result from cultural norms and the healthcare systems available in a particular country; individual healthrelated beliefs and psychosocial factors also play a role $(17,18)$. To-date, only one study reported on insulin refusal among the Korean population, which, over a decade ago, was estimated at $67 \%(10)$. Nevertheless, this study had several limitations. First, the sample size was relatively small $(\mathrm{N}=76)$. Second, PIR was assessed with an invalid instrument. In addition, it should be noted that healthcare policy on chronic disease management in Korea has changed significantly over the past 10 years; as such, attitudes and beliefs toward insulin, and thus the insulin refusal rate, are likely to have changed. Since the publication of the first study of insulin refusal among Koreans, the data regarding PIR in this population has not been updated and the impact of the psychosocial burden of diabetes on insulin therapy uptake remains incompletely understood. To fill this gap, this study examined the insulin refusal rate and PIR level among Korean patients with type 2 diabetes.

\section{Methods}

\section{Study design}

This was a cross-sectional study to describe patient intentions to receive insulin therapy and their PIR levels. We compared PIR levels in the insulin refusals among the groups intention to receive insulin therapy after being recommended for insulin therapy.

\section{Study sample}

Patients with type 2 diabetes, registered at the endocrinology departments of two university hospitals and two public healthcare centers in an urban area of Korea, participated in this study; data were collected between January and December in 2016. Required sample size to compare the PIR level among groups was calculated based on the prior study (10) using $G^{*}$ Power 3.1 (www.gpower. hhu.de), applying an effect size of 0.25 (medium), power (1- $\beta$ ) 0.80 , and an $\alpha$ of 0.05 for one way ANOVA analysis. The required sample size was 73 , a total of 515 patients were satisfied this requirement in this study. However, we tried to include as many eligible participants as possible to investigate the insulin refusal rates. Individuals were eligible for inclusion in the study if they were diagnosed with type 2 diabetes, recommended insulin therapy, and $\geq 19$-yearold. Participants were excluded from the study if they were diagnosed with a psychological disorder or cognitive impairment, such as dementia, if they were hospitalized during the study period, or diagnosed with type 1 or gestational diabetes.

Patients provided written informed consent during their scheduled follow-up appointments after receiving an explanation of the purpose and procedure of the study (including the voluntary nature of the study, the right to withdraw, and confidentiality). Trained research staff administered the questionnaire during a face-to-face interview at a private outpatient office. After completing the questionnaire, prescriptions were checked to confirm the treatment type (i.e., oral agents, injections) recommended to each patient.

The study protocol was approved by the appropriate institutional review boards (No.: 2-1046881-A-N-01201410-HR-046 and CNUH 2015-09-005).

\section{Measurements}

\section{Insulin refusal}

Insulin refusal was determined using the following questions: "Have you been recommended for insulin therapy by a physician?" and "If you answered yes, are you taking insulin therapy, as recommended?" Participants who answered "yes" (taking insulin therapy) were grouped as G1 (insulin acceptance); participants who answered "no", were grouped as G2 (insulin refusal). Trained research staff reviewed each participant's medical records to verify whether the patient had rejected insulin therapy. The prevalence of insulin refusal was calculated as the ratio of the number of patients who refused insulin (G2) over the total number of patients recommended for insulin therapy $(\mathrm{G} 1+\mathrm{G} 2)$. 
Table 1 Prevalence of insulin refusal $(\mathrm{N}=515)$

\begin{tabular}{|c|c|c|c|}
\hline Recommendations of insulin therapy & \multicolumn{2}{|c|}{ Insulin therapy } & Insulin refusal rate (\%) \\
\hline Yes (n, \%) & G1: 331 (64.3) & G2: $184(35.7)$ & {$[\mathrm{G} 2 /(\mathrm{G} 1+\mathrm{G} 2)] \times 100=35.7 \%$} \\
\hline
\end{tabular}

G1, group: insulin acceptance group; G2, group: insulin refusal group.

\section{Intention to receive insulin therapy}

Only the G2 group patients were asked about their intention to receive insulin therapy. The following question was used: "Do you intend to take insulin, as recommended?" This was a multiple-choice question with available answers as follows: "Not necessary (recognized as insulin denial)," "Would like to delay insulin therapy as long as I can," and "Will receive within 3 montbs."

\section{PIR}

PIR was measured using the Korean version of the Psychological Insulin Resistance scale (K-PIR), which was developed and validated by Song et al. (3). The K-PIR consists of two subscales that include psycho-cognitive (negative feelings, low awareness, low confidence in being able to self-inject, dependent lifestyle, and embarrassment) and supportive (economic burden and feeling about family/ friends) factors. The 18 items included in the scale were evaluated using a five-point Likert scale to yield a total score of $18-90$, with higher scores indicating a higher level of PIR. In a study conducted by Song et al., Cronbach's alpha was 0.90 for the psycho-cognitive factors and 0.74 for supportive factors (3); in this study, Cronbach's alpha was 0.90 and 0.76 , respectively.

\section{Co-morbidity}

Co-morbidities included chronic conditions other than diabetes or its complications, such as cardiac disease, hypertension, cerebral vascular disease, renal disease, retinopathy, neuropathy, gastrointestinal disease, and foot ulcers.

\section{Data analysis}

The insulin refusal rate was calculated based on the number of participants who did not take insulin therapy (G1) over the total number of patients who were recommended insulin therapy (G2). Independent t-test and chi-square tests were performed to compare the distribution of patient demographic characteristics between G1 (insulin acceptance) and G2 (insulin refusal) groups. The degree of PIR in the insulin refusal group was examined using ANOVA and a post-hoc Scheffe test. $\mathrm{P}$ values $<0.05$ were considered statistically significant.

\section{Results}

\section{Prevalence of insulin refusal}

Table 1 presents the prevalence of insulin refusal. The insulin refusal rate was $35.7 \%$ (the percentage of insulin-refusal patients $(\mathrm{G} 2, \mathrm{n}=184)$ among all patients recommended to undergo insulin therapy by their health care providers (G1 $+\mathrm{G} 2, \mathrm{n}=515)$.

\section{Demographic characteristics of patients accepting and refusing insulin therapy}

Table 2 shows the socio-demographic characteristics of the patients in the two groups. Gender $\left(x^{2}=0.02, \mathrm{P}=0.927\right)$, having a spouse $\left(x^{2}=0.75, \mathrm{P}=0.398\right)$, religion $\left(x^{2}=0.25\right.$, $\mathrm{P}=0.644)$, educational level $\left(x^{2}=7.63, \mathrm{P}=0.054\right)$, satisfaction with economic status $\left(x^{2}=0.47, \mathrm{P}=0.516\right)$, and mean age $(\mathrm{t}=-0.39, \mathrm{P}=0.691)$ were not significantly different between the groups. However, significant differences were found in having received insulin education $\left(x^{2}=122.29, \mathrm{P}<0.001\right)$, duration of diabetes $(\mathrm{t}=-5.60, \mathrm{P}<0.001)$, number of comorbid conditions ( $\mathrm{t}=-2.13, \mathrm{P}=0.034)$, and glycosylated hemoglobin (HbA1c) level $(\mathrm{t}=-2.40, \mathrm{P}=0.017)$.

\section{Intention to receive insulin therapy among the insulin refusal group}

Among the G2 group, the rates of patients responding with "Not necessary (insulin denial)" or "Want to delay as long as I can" were $63.6 \%(\mathrm{n}=117)$ and $7.1 \%(\mathrm{n}=13)$, respectively; $29.3 \%(\mathrm{n}=54)$ were willing to receive insulin therapy within 3 months (Table 3).

The total mean PIR score was significantly lower for patients willing to receive insulin therapy within 3 months 
Table 2 Comparisons of characteristics between Insulin-acceptance and refusal groups

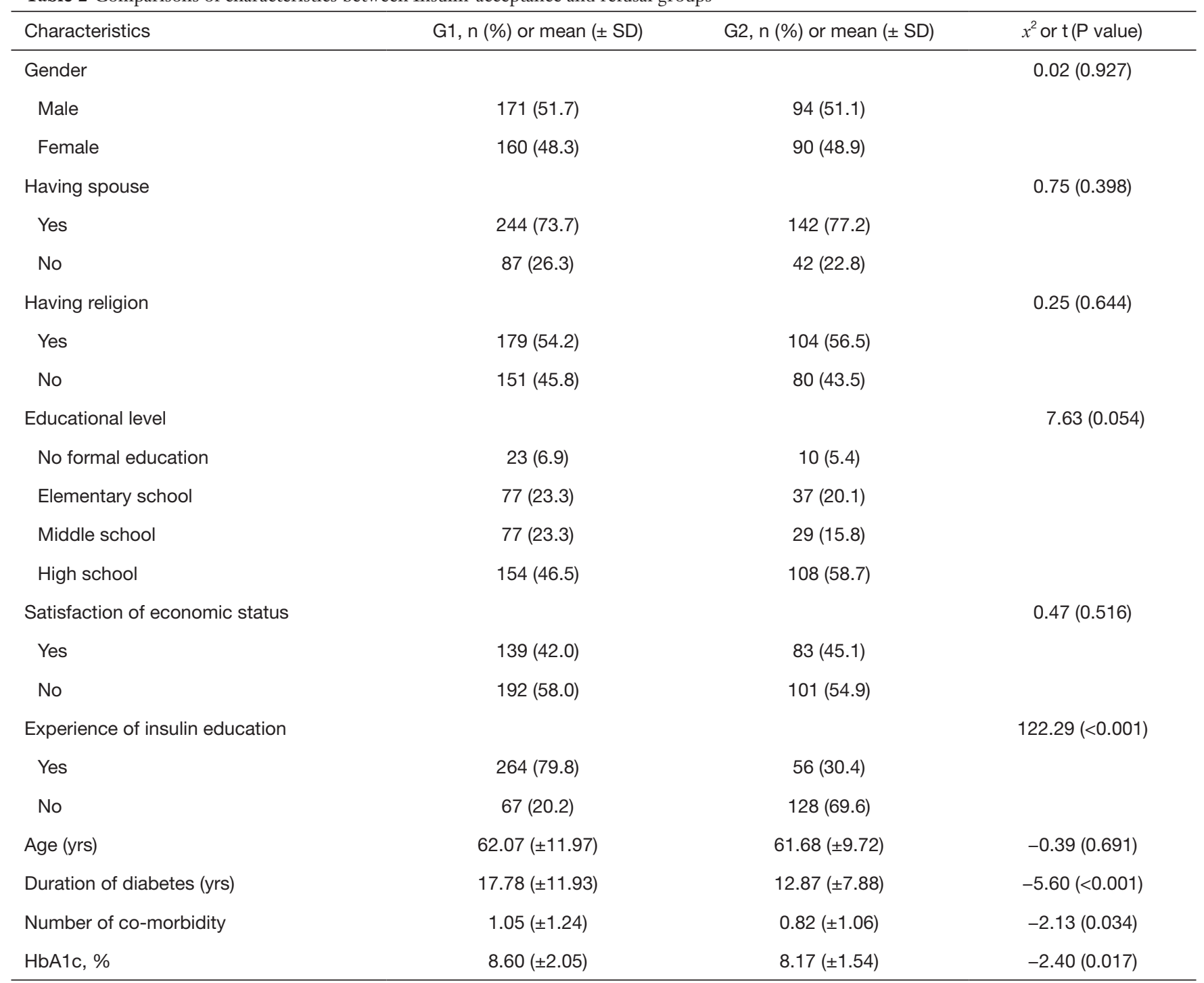

G1, group 1= Insulin-acceptance group; G2, group 2= Insulin-refusal group; HbA1c, serum hemoglobin A1c; Number of co-morbidity, cardiac disease, hypertension, cerebral vascular disease, renal disease, retinopathy, neuropathy, gastrointestinal disease, foot ulcers.

Table 3 Intention to receive insulin therapy and psychological insulin resistance in the insulin refusal group (N=184)

\begin{tabular}{lcccc}
\hline PIR & $\begin{array}{c}\text { Unnecessary } \\
\text { (insulin denial) (a) }\end{array}$ & $\begin{array}{c}\text { Want to delay as } \\
\text { long as I can (b) }\end{array}$ & $\begin{array}{c}\text { Will receive within } \\
3 \text { months }(\mathrm{c})\end{array}$ & $\mathrm{F}(\mathrm{p}) / \mathrm{Scheffe}$ post hoc test \\
\hline $\mathrm{n}(\%)$ & $117(63.6)$ & $13(7.1)$ & $54(29.3)$ & \\
Total PIR, mean $( \pm \mathrm{SD})$, range: $18-90$ & $64.69( \pm 13.93)$ & $60.69( \pm 11.46)$ & $47.81( \pm 14.70)$ & $26.83(0.001) ;(\mathrm{c})<(\mathrm{a}),(\mathrm{b})$ \\
Psycho-cognitive factor, mean $( \pm \mathrm{SD})$, range: $14-70$ & $51.72( \pm 11.07)$ & $47.76( \pm 8.88)$ & $36.94( \pm 12.08)$ & $31.91(0.001) ;(\mathrm{c})<(\mathrm{a}),(\mathrm{b})$ \\
Supportive factor, mean $( \pm \mathrm{SD})$, range: 4-20 & $12.96( \pm 4.19)$ & $12.92( \pm 3.90)$ & $10.87( \pm 4.17)$ & $4.80(0.009) ;(\mathrm{c})<(\mathrm{a})$ \\
\hline
\end{tabular}

PIR, psychological insulin resistance. 
$(\mathrm{F}=26.83, \mathrm{P}<0.001)$ than for individuals in the "insulin unnecessary (insulin denial)" and "want to delay" groups. The mean scores for the psycho-cognitive $(\mathrm{F}=31.91$, $\mathrm{P}<0.001)$ and supportive $(\mathrm{F}=4.80, \mathrm{P}=0.009)$ factors were highest for patients in the insulin refusal group who answered, "Not necessary (insulin denial)."

\section{Conclusions}

This study showed that the prevalence of insulin refusal among Korean patients with type 2 diabetes is $35.7 \%$, which is similar to the results of previous studies involving nonAsian populations $(4,7)$. Larkin et al. reported that one-third of insulin-refusal patients with type 2 diabetes expressed an unwillingness to take prescribed insulin (7). However, when compared with other studies of Asian populations with type 2 diabetes, the insulin therapy refusal rate in the present study was relatively low. Recent studies conducted in Asian populations have shown that about $40-70 \%$ of type 2 diabetes patients in Singapore (6) and Pakistan (9) refused insulin therapy.

Over the last decade, in Korea, the prevalence of diabetes has rapidly increased to $12.4 \%$ in 2011 , compared to $9.1 \%$ in 2005; a further 2-fold increase is expected over the next 40 years (16). Despite the growing prevalence of diabetes, insulin refusal rate has declined from $67 \%$ in 2008 (10) to $35.7 \%$ in the current study. This decrease coincides with efforts to reduce the prevalence of diabetes in Korea that have been taking place since 2008. Health policies have emphasized the importance of following diabetes treatment guidelines and have encouraged clinicians to identify patients needing insulin therapy. For example, patients with type 2 diabetes requiring insulin may now receive insulin therapy education from advanced medical institutes, with support from Korea's national health insurance. Moreover, since 2011, several regional public healthcare centers have implemented diabetes registration and control programs for older adults, with support from the Korea Centers for Disease Control \& Prevention (19), and the recommendation of insulin pen uses by physicians. These activities may have influenced the prevalence of insulin refusal.

Although the rate of insulin acceptance has increased, the PIR levels among insulin-refusal patients remain similar to those observed in a previous study (10). This demonstrates that the perceived psychological barriers to insulin therapy initiation and adherence among insulin-refusing patients have not changed, despite implementation of a diabetes control program in Korea in 2011. In the Diabetes
Attitudes, Wishes, and Needs (DAWN) study, conducted in 13 countries, the 5As (assess-advise-agree-assist-arrange) program was developed to identify the precise barriers and sources of concern for individuals with PIR (20). The 5 As program recommended specific approaches to address insulin use barriers (20), but most of the recommended strategies involved explaining the reasons for prescribing insulin and teaching the patient how to simplify insulin administration. Some studies reported that PIR can be influenced by culture, age, sex, and socioeconomic status (11), as well as linked with individual psychological states, such as depression and distress (21). As such, educational approaches focused on the medical aspect of insulin therapy alone may be limited in their ability to reduce PIR among some insulin-naïve patients. Luk stated that 'healthcare providers should be alerted to the multi-dimensional nature of PIR' (21). Nevertheless, the need remains for further comprehensive research to better understand the factors related to PIR and how to address them with patients.

In this study, disease duration and the number of diabetes-associated complications were statistically different between insulin-accepting and insulin-refusing groups. Similar to the previous studies, the mean age of patients was lower in the insulin-refusing than insulin-accepting groups, but this difference was not significant in this study. In a study by Machinani et al., lower mean age and lower levels of education were found in insulin-refusing respondents than insulin-accepting respondents (11). In the present study, longer disease duration, greater numbers of comorbidities, and uncontrolled HbAlc levels were found among the insulin-refusing group than insulin-accepting group, which was consistent with previous studies $(12,22,23)$. In the present study, a significantly higher number of patients in the insulin-accepting group had experienced insulin education, compared with the insulin-refusing group. This result provides additional evidence that diabetes management education can influence a patient's decision to change their insulin-related behavior.

Some limitations were present in this study. First, the patients involved in this study were from two cities in Korea, which might limit the generalizability of the findings. Future research should include larger samples across multiple research sites. Second, reluctance toward insulin was investigated through a self-report and confirmed with a medical record check, which is associated with a risk of patients concealing their real attitudes toward insulin and some records might have been omitted. As a result, insulin refusal rate reported in this might be subject to bias. Third, 
the PIR measurement tool used in this study, the K-PIR tool, may also present a limitation. Although the K-PIR was developed from existing instruments, including the Barriers to Insulin Treatment questionnaire and the Problem Areas in Diabetes survey, the simple comparison of PIR levels between other ethnic groups and Koreans may be a limitation. Further studies are needed to analyze the differences in PIR levels between Asian and non-Asian populations, using the same tool. As an example, the DAWN study used specific tools to compare various psychosocial outcomes, such as quality of life and diabetes treatment burden, in 17 countries (18). Moreover, reasons for PIR in patients should be investigated using in-depth interviews for better understanding of the motivations driving each patient's decision-making.

Despite these potential limitations, this study was, to the best of our knowledge, it attempts to explore various aspects of insulin refusal and PIR in Korean patients with type 2 diabetes. Several important clinical implications for the care of Koreans with type 2 diabetes can be addressed. As previously emphasized in the literature, PIR should be continuously monitored among all patients with diabetes, and appropriate interventions should be applied. Healthcare providers should understand that psychological resistance to insulin therapy is a continuous process experienced by patients with diabetes. Furthermore, healthcare providers should respect patients' decisions, while also planning individualized education and counseling regarding insulin use and other self-management techniques implemented in healthcare settings. This study found that the prevalence of insulin resistance has decreased in Korea over the past decade, but the PIR level in the insulin-naïve group has remained unchanged. As national policies and support may affect insulin refusal rates, economic support and the national insurance system should be further developed to reduce resistance to insulin therapy among Korea patients with type 2 diabetes.

\section{Acknowledgments}

Funding: This work was supported by the National Research Foundation of Korea (NRF) grant funded by the Korea government (Ministry of Science and ICT, MSIT) (NRF2019R1A2C1006016).

\section{Footnote}

Conflicts of Interest: The authors have no conflicts of interest to declare.
Ethical Statement: The authors are accountable for all aspects of the work in ensuring that questions related to the accuracy or integrity of any part of the work are appropriately investigated and resolved. The study protocol was approved by the appropriate institutional review boards (No. 2-1046881-A-N-01-201410-HR-046 and CNUH 2015-09-005).

\section{References}

1. American Diabetes Association. 9. Pharmacologic Approaches to Glycemic Treatment: Standards of Medical Care in Diabetes-2019. Diabetes Care 2019;42:S90-102.

2. Brod M, Kongsø JH, Lessard S, et al. Psychological insulin resistance: patient beliefs and implications for diabetes management. Qual Life Res 2009;18:23-32.

3. Song Y, Jeon Y, Cho J, et al. Development of a psychological insulin resistance scale for Korean patients with diabetes. J Korean Acad Nurs 2016;46:813-23.

4. Polonsky WH, Fisher L, Guzman S, et al. Psychological insulin resistance in patients with type 2 diabetes: the scope of the problem. Diabetes Care 2005;28:2543-5.

5. Nam S, Nam S, Song Y. Role of self-efficacy in the relationship between patient-provider relationships and psychological insulin resistance among patients with type 2 diabetes. J Contemp Diabetes Res 2014;1:1-15.

6. Wong S, Lee J, Ko Y, et al. Perceptions of insulin therapy amongst Asian patients with diabetes in Singapore. Diabet Med 2011;28:206-11.

7. Larkin ME, Capasso VA, Chen CL, et al. Measuring psychological insulin resistance: barriers to insulin use. Diabetes Educ 2008;34:511-7.

8. Khan H, Lasker SS, Chowdhury TA. Prevalence and reasons for insulin refusal in Bangladeshi patients with poorly controlled type 2 diabetes in East London. Diabet Med 2008;25:1108-11.

9. Saleem A, Masood I, Khan TM, et al. Insulin perception among insulin-refusal type-2 diabetes mellitus patients in Pakistan. Cogent Med 2016;3:1229374.

10. Hong SH, Kim MJ, Noh SG, et al. A Study on Resistance in Type 2 Diabetic Patient Against Commencement of Insulin Treatment. Korean Diabetes J 2008;32:269-79.

11. Machinani S, Bazargan-Hejazi S, Hsia SH. Psychological insulin resistance among low-income, U.S. racial minority patients with type 2 diabetes. Prim Care Diabetes 2013;7:51-5.

12. Nur Azmiah Z, Zulkarnain AK, Aris T. Psychological insulin resistance (PIR) among type 2 diabetes patients 
at public health clinics in Federal Territory of Malaysia. International Medical Journal Malaysia 2011;10:7-12

13. Nam S, Chesla C, Stotts NA, et al. Factors associated with psychological insulin resistance in individuals with type 2 diabetes. Diabetes Care 2010;33:1747-9.

14. Nam S, Song HJ, Park SY, et al. Challenges of diabetes management in immigrant Korean Americans. Diabetes Educ 2013;39:213-21.

15. Song Y, Song HJ, Han HR, et al. Unmet needs for social support and effects on diabetes self-care activities in Korean Americans with type 2 diabetes. Diabetes Educ 2012;38:77-85.

16. Song Y, Ah E. Patients' perspectives on taking insulin in diabetes - Perspectives of convergence. J Digit Converg 2016;14:283-92.

17. Ng CJ, Lai PS, Lee YK, et al. Barriers and facilitators to starting insulin in patients with type 2 diabetes: a systematic review. Int J Clin Pract 2015;69:1050-70.

18. Nicolucci A, Kovacs Burns K, Holt RI, et al. Diabetes Attitudes, Wishes and Needs second study (DAWN2 ${ }^{\mathrm{TM}}$ ):

Cite this article as: Song Y, Ku BJ, Cho J, Jun Y, Kim B, Nam $\mathrm{S}$. The prevalence of insulin refusal and psychological insulin resistance among Korean patients with type 2 diabetes mellitus. Ann Transl Med 2019;7(23):760. doi: 10.21037/atm.2019.11.77
Cross-national benchmarking of diabetes-related psychosocial outcomes for people with diabetes. Diabet Med 2013;30:767-77.

19. Korean Center for Disease Prevention. Division of chronic disease control. Available online: http://www. cdc.go.kr/CDC/eng/contents/CdcEngContentView. jsp?cid=17909\&menuIds=HOME002-MNU0575MNU0634. Accessed 30 July 2018.

20. Peyrot M, Rubin RR, Khunti K. Addressing barriers to initiation of insulin in patients with type 2 diabetes. Prim Care Diabetes 2010;4:S11-8.

21. Luk A. Psychological insulin resistance: scope of the problem. Hong Kong Med J 2016;22:304-5.

22. Bahrmann A, Abel A, Zeyfang A, et al. Psychological insulin resistance in geriatric patients with diabetes mellitus. Patient Educ Couns 2014;94:417-22.

23. Weinger K, Beverly EA. Barriers to achieving glycemic targets: who omits insulin and why? Diabetes Care 2010;33:450-2. 